\title{
Optimized Pulse Patterns for Salient Synchronous Machines
}

\author{
Nina Hartgenbusch*a) Non-member, Anna Thünen** Non-member \\ Rik W. De Doncker* Non-member
}

(Manuscript received January 4, 2021)

\begin{abstract}
Synchronous optimal pulse width modulation is commonly applied when the ratio between the fundamental frequency and the switching frequency is low. Offline computed pulse patterns are optimized in terms of the harmonic distortion in phase currents. Typically, the underlying optimization problem is based on a simple inductor model representing the harmonic behavior of one phase of a nonsalient machine. This paper proposes an alternative formulation of the optimization problem, which generalizes it for salient machine types. An investigation of the optimization results' sensitivity to operating point-dependent parameters is presented.
\end{abstract}

Keywords: Interior Permanent Magnet Synchronous Machines, Pulse-Width Modulation, Optimized Pulse Patterns

\section{Nomenclature}

$x_{\mathrm{s}} \quad$ Stator quantity

$\underline{x} \quad$ Complex quantity

$\overline{\hat{x}} \quad$ Magnitude of $x$

$x^{\mathrm{dq}} \quad$ Space vector in synchronous dq-frame

$\vec{x}^{\alpha \beta} \quad$ Vector in $\alpha \beta$-frame

$x^{\mathrm{a}}, x^{\mathrm{b}}, x^{\mathrm{c}} \quad$ Phase quantities of a symmetric three phase system

$\omega_{1} \quad$ Fundamental electrical angular frequency

$k \quad$ Order of the harmonic component at the frequency $k \omega_{1}$

$u_{\mathrm{s}}^{\mathrm{d}}, u_{\mathrm{s}}^{\mathrm{q}} \quad$ Voltages in synchronous dq-frame (fundamental component)

$i_{\mathrm{s}}^{\mathrm{d}}, i_{\mathrm{s}}^{\mathrm{q}} \quad$ Currents in synchronous dq-frame (fundamental component)

$\theta_{\mathrm{dq}} \quad$ Angle between the fundamental voltage space vector and the $d$-axis of the synchronous reference frame

$i_{\mathrm{s}, \mathrm{u} k} \quad$ Current caused by a harmonic voltage $u_{k}$

$i_{\mathrm{s}, k} \quad$ Current of harmonic order $k$

$L_{\mathrm{sd}}, L_{\mathrm{sq}} \quad$ Inductances in synchronous dq-frame

X Saliency $\frac{L_{\mathrm{sq}}}{L_{\mathrm{sd}}}$

$R_{\mathrm{s}} \quad$ Stator resistance

$\psi_{\mathrm{f}} \quad$ Field flux linkage

a) Correspondence to: Nina.Hartgenbusch@ isea.rwth-aachen.de

${ }^{*}$ Institute for Power Electronics and Electrical Drives (ISEA)

RWTH Aachen University

Jägerstrasse 17/19 - 52066 Aachen - Germany

Email: post@isea.rwth-aachen.de

** Institute for Geometry and Pratical Mathematics (IGPM)

RWTH Aachen University

Templergraben 55 - 52056 Aachen - Germany

This publication is based on ${ }^{(1)}$, which was published in the Proceedings of the International Conference on Electrical Machines and Systems 2020 CIEEE

\begin{tabular}{|c|c|}
\hline$U_{\mathrm{dc}}$ & Dc-link voltage \\
\hline$m$ & Modulation index defined as $m=\frac{\hat{u}_{\mathrm{s}, 1}}{U_{\mathrm{dc}}}$ \\
\hline$d$ & Distortion \\
\hline$M$ & $\begin{array}{l}\text { Number of adjustable switching angles within } \\
\text { one quarter period }\end{array}$ \\
\hline$\alpha$ & $\begin{array}{l}\text { Vector of switching angles } \boldsymbol{\alpha}=\left(\alpha_{1}, \ldots, \alpha_{M}\right) \\
\text { which are the decision variables in the opti- } \\
\text { mization }\end{array}$ \\
\hline
\end{tabular}

\section{Introduction}

Due to their high efficiency and good power density, permanent magnet synchronous machines recently became more attractive also for high power traction drives. In mediumvoltage drive applications the switching frequency of the inverter is often limited to several $100 \mathrm{~Hz}$. At high electrical frequencies, the ratio between the fundamental and the switching frequency becomes so low that it is reasonable to apply synchronous pulse width modulation. The offline computation of synchronous optimized pulse patterns (OPPs) is a well-known approach to minimize the total harmonic distortion (THD) of the phase currents, first presented in ${ }^{(2)}$.

Most publications related to OPPs derive the optimization problem based on the harmonic equivalent circuit of an induction machine, e.g. ${ }^{(3)(6)}$. $\operatorname{In}^{(7)}$, the conventional approach is applied to a nonsalient permanent magnet synchronous machine (PMSM) and in ${ }^{(8)}$ the saliency of the PMSM is even neglected. This way, the complexity of the underlying optimization problem can be reduced tremendously using a cost function which is independent of any machine parameters.

However, this approach is not physically valid for machines with saliency like interior permanent magnet synchronous machines. A first calculation approach suited for salient-pole synchronous machines has been presented in ${ }^{(9)}$. Still, the dependency of the optimized switching angles on operating point dependent parameters apart from the modu- 


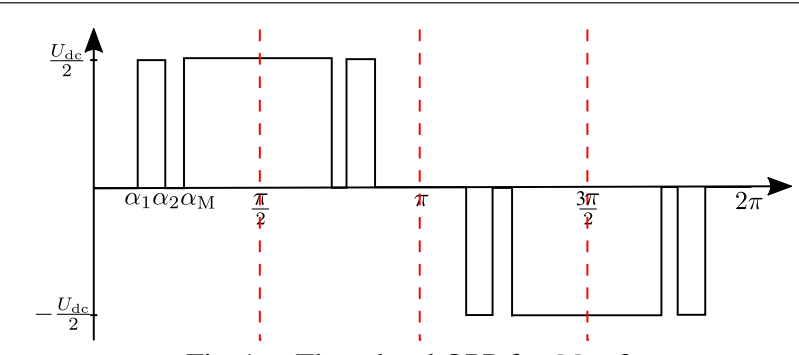

Fig. 1. Three level OPP for $M=3$.

lation index is not considered in ${ }^{(9)}$.

This paper provides an insight in the coupling between related harmonics which is caused by anisotropic magnetic properties. Based on these relations, a formulation of the optimization problem suited for salient synchronous machines is presented and used to evaluate the impact of the operating point dependent parameters on the optimization results.

\section{Cross coupling between harmonic phase cur- rents}

First, the voltage and the current expressions are derived for a three-level converter topology. An exemplary voltage form is shown in figure 1.

The optimized switching angles are denoted by $\alpha_{i}$ with $i \in$ $[1, M]$ where $M$ is the number of switching events per quarter cycle which is equivalent to the number of variables in the optimization. Only the odd, nontriplen order harmonics remain for an Y-connection of the phases and quarter wave symmetry, cf. ${ }^{(10)}$. Denote these harmonics by $k=6 n \pm 1$ for $n=1,2,3, \ldots$. The harmonic voltage magnitude at the inverter terminals may be formulated with Fourier decomposition as:

$$
\hat{u}_{\mathrm{s}, k}(\boldsymbol{\alpha})=\frac{2 U_{\mathrm{dc}}}{\pi} \frac{1}{k} \sum_{i=1}^{M}(-1)^{i+1} \cos \left(k \alpha_{i}\right) .
$$

For the derivation of the optimization problem, an appropriate representation of the harmonic phase voltages and currents is necessary.

Within this work, a standard linear model of a synchronous machine in synchronous dq-frame is considered. The respective voltage equations for the fundamental components are stated in 2.

$$
\begin{aligned}
& u_{\mathrm{s}}^{\mathrm{d}}=R_{\mathrm{s}} i_{\mathrm{s}}^{\mathrm{d}}+L_{\mathrm{sd}} \cdot \frac{d i_{\mathrm{s}}^{\mathrm{d}}}{d t}-\omega_{1} L_{\mathrm{sq}} i_{\mathrm{s}}^{\mathrm{q}} \\
& u_{\mathrm{s}}^{\mathrm{q}}=R_{\mathrm{s}} i_{\mathrm{s}}^{\mathrm{q}}+L_{\mathrm{sq}} \cdot \frac{d i_{\mathrm{s}}^{\mathrm{q}}}{d t}+\omega_{1} L_{\mathrm{sq}} i_{\mathrm{s}}^{\mathrm{q}}+\omega_{1} \psi_{\mathrm{f}}
\end{aligned}
$$

The following simplifications have been carried out:

- The back emf voltage $u_{\mathrm{emf}}=\omega_{1} \cdot \psi_{\mathrm{f}}$ is assumed to be perfectly sinusoidal.

- For the consideration of the current harmonics, the resistive part of the impedance is neglected since $R_{\mathrm{S}} \ll(k-1) \omega_{1} L_{\mathrm{s}}$ for higher order harmonics.

- Other design specific sources of harmonic phase current, namely spatial harmonics, which are mostly dependent

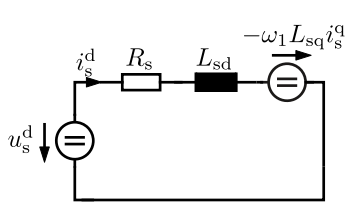

(a) fundamental d components

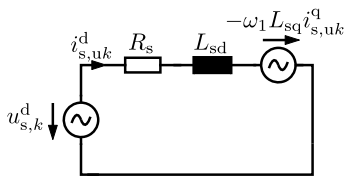

(c) harmonics d components

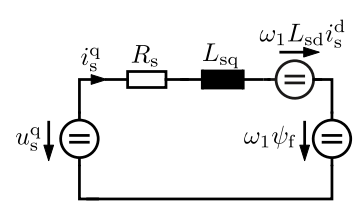

(b) fundamental q components

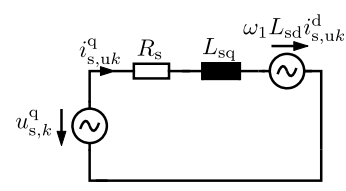

(d) harmonics q components
Fig. 2. Equivalent circuit diagrams in synchronous reference frame for a salient permanent magnet synchronous machine.

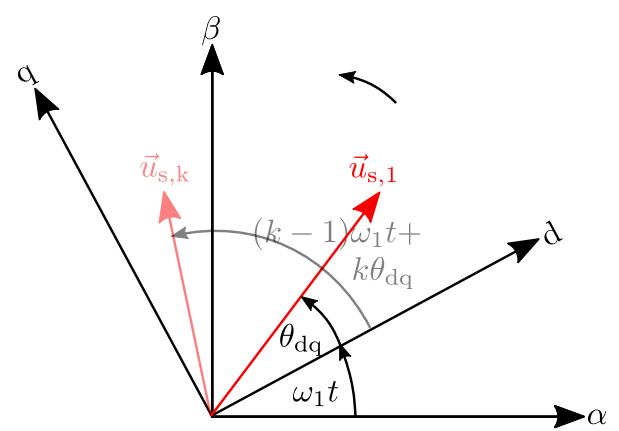

Fig. 3. The fundamental and a positive sequence harmonic voltage space vector in synchronous dq-frame.

on the winding configuration and slotting are not taken into account.

The corresponding equivalent circuit diagrams for both fundamental d- and q-components are shown in the figures $2 \mathrm{a}$ and $2 \mathrm{~b}$. Whereas the fundamental voltage and current components transform into dc quantities, the harmonic components are still ac quantities in dq-frame as depicted in the figures $2 \mathrm{c}$ and $2 \mathrm{~d}$.

The angular velocity of a harmonic space vector rotating in the synchronous dq-frame can be determined by the transformation $\vec{x}^{\mathrm{dq}}=\vec{x}^{\alpha \beta} \cdot \mathrm{e}^{-j \omega_{1} t}$. As illustrated in figure 3, a space vector of the harmonic order $k$ rotates with an angular velocity of $(k-1) \omega_{1}$ in the dq-frame. It is crucial to distinguish between positive and negative sequence harmonics. A space vector related to a phase voltage of harmonic order $k=6 n+1$ is denoted as positive sequence harmonic and rotates with $(6 n+1) \omega_{1}$ in the $\alpha \beta$-frame or with $6 n \omega_{1}$ in the dq-frame. On the contrary, a phase voltage of harmonic order $k=6 n-1$ is referred to a negative sequence harmonic and rotates with $-(6 n-1) \omega_{1}$ in the $\alpha \beta$-frame or with $-6 n \omega_{1}$ in the dq-frame.

An arbitrary harmonic voltage space vector $\vec{u}_{\mathrm{s}, k}^{\mathrm{dq}}$ with $\hat{u}_{\mathrm{s}, \mathrm{k}}=\left|\vec{u}_{\mathrm{s}, k}\right| \mathrm{dq} \mid$ of order $k=6 n \mp 1$ can be expressed as:

$$
\begin{aligned}
\vec{u}_{\mathrm{s}, k}^{\mathrm{dq}}= & \hat{u}_{\mathrm{s}, \mathrm{k}} \cdot \mathrm{e}^{\mathrm{j}\left(\mp(k-1) \omega_{1} t \mp k \theta_{\mathrm{dq}}+\frac{\pi}{2} \mp \frac{\pi}{2}\right)} \\
= & \hat{u}_{\mathrm{s}, \mathrm{k}} \cdot\left[\cos \left(\mp(k-1) \omega_{1} t \mp k \theta_{\mathrm{dq}}+\frac{\pi}{2} \mp \frac{\pi}{2}\right)\right. \\
& \left.+\mathrm{j} \cdot \sin \left(\mp(k-1) \omega_{1} t \mp k \theta_{\mathrm{dq}}+\frac{\pi}{2} \mp \frac{\pi}{2}\right)\right] .
\end{aligned}
$$

Note that the harmonic voltage expression in (3) is depen- 
dent on the angle $\theta_{\mathrm{dq}}$ (defined in figure 3 ). This can be interpreted as a phase shift of the fundamental voltage component which is dependent on the operating point as well as the chosen operating trajectory. The d- and q-component of $\vec{u}_{\mathrm{s}, k}^{\mathrm{dq}}$ in figure 3 can be extended to complex quantities in order to facilitate the further calculations. The corresponding complex vectors are stated as:

$$
\begin{aligned}
\underline{u}_{\mathrm{s}, k}^{\mathrm{d}} & =\hat{u}_{\mathrm{s}, \mathrm{k}} \cdot \mathrm{e}^{\mathrm{j}\left(\mp(k-1) \omega_{1} t \mp k \theta_{\mathrm{dq}}+\frac{\pi}{2} \mp \frac{\pi}{2}\right)}, \\
\underline{u}_{\mathrm{s}, k}^{\mathrm{q}} & =\hat{u}_{\mathrm{s}, \mathrm{k}} \cdot \mathrm{e}^{\mathrm{j}\left(\mp(k-1) \omega_{1} t \mp k \theta_{\mathrm{dq}}+\frac{\pi}{2} \mp \frac{\pi}{2}-\frac{\pi}{2}\right)}, \\
& =-\mathrm{j} \cdot \underline{u}_{\mathrm{s}, k}^{\mathrm{d}} .
\end{aligned}
$$

Considering the circuits depicted in figures $2 \mathrm{c}$ and $2 \mathrm{~d}$, with $R_{\mathrm{S}} \ll(k-1) \omega_{1} L_{\mathrm{S}}$ the currents evoked by a harmonic voltage of order $k$ are written in complex form as follows:

$$
\begin{aligned}
& \underline{i}_{-\mathrm{s}, \mathrm{u} k}^{\mathrm{d}}=\frac{\underline{u}_{\mathrm{s}, k}^{\mathrm{d}}+\omega_{1} L_{\mathrm{sq}} \underline{\mathrm{i}}_{\mathrm{s} \mathrm{u} k}^{\mathrm{q}}}{\mathrm{j} \cdot \mp(k-1) \omega_{1} L_{\mathrm{sd}}}, \\
& \underline{i}_{\mathrm{s}, \mathrm{u} k}^{\mathrm{q}}=\frac{\underline{u}_{\mathrm{s}, k}^{\mathrm{q}}-\omega_{1} L_{\mathrm{sd}} \underline{\mathrm{d}}_{\mathrm{s}, \mathrm{u} k}^{\mathrm{d}}}{\mathrm{j} \cdot \mp(k-1) \omega_{1} L_{\mathrm{sq}}} .
\end{aligned}
$$

Rearranging the equations (5) yields expressions depending only on the applied voltages which are deduced by equations (1) and (4). The magnitudes of the phase currents in dq-domain $\hat{i}_{\mathrm{s}, \mathrm{u} k}^{\mathrm{d}}$ and $\hat{i}_{\mathrm{s}, \mathrm{u} k}^{\mathrm{g}}$ are determined by the absolute value of the complex vectors $\underline{i}_{\mathrm{s}, \mathrm{u} k}^{\mathrm{d}}$ and $\underline{i}_{\mathrm{s}, \mathrm{u} k}^{\mathrm{q}}$.

In the following, a small-signal analysis is carried out to illustrate the coupling behavior between related harmonics of order $k=6 n \pm 1$. For this purpose, a simulation with a 5th order harmonic voltage applied to the machine is performed. The respective low-pass filtered steady-state voltages and phase currents are depicted in figure 4 . There, it is visible that a harmonic voltage leads to an ac current with different magnitudes in dq-domain which is caused by the different impedances due to $L_{\mathrm{sd}} \neq L_{\mathrm{sq}}$, cf. (5). Note that in case of non-salient machines, $L_{\mathrm{sd}}=L_{\mathrm{sq}}$ leads to $\hat{i}_{\mathrm{s}, \mathrm{u} k}^{\mathrm{d}}=$ $\hat{i}_{\mathrm{s}, \mathrm{u} k}^{\mathrm{q}}$. The corresponding phase current $i_{\mathrm{s}}^{\mathrm{a}}$ exhibits the form of an envelope signal. The spectrum of this envelope signal reveals that the phase current contains in addition to a component of $k=6 n-1=5$ also one of the related harmonic $k=6 n+1=7$. A similar behavior is observable for an applied voltage of order $k=6 n+1$ which causes an additional phase current component of order $k=6 n-1$.

The simple mathematical proof of this behavior is provided in the following. An arbitrary positive sequence harmonic current vector in dq-frame which is caused by harmonic voltage of order $k=6 n+1$ may be expressed as:

$$
\begin{aligned}
\vec{i}_{\mathrm{s}, \mathrm{u} k}^{\mathrm{dq}}= & \hat{i}_{\mathrm{s}, \mathrm{u} k}^{\mathrm{d}} \cos \left(6 n \omega_{1} t\right)+\mathrm{j} \cdot \hat{i}_{\mathrm{s}, \mathrm{u} k}^{\mathrm{q}} \sin \left(6 n \omega_{1} t\right) \\
= & \hat{i}_{\mathrm{s}, \mathrm{u} k}^{\mathrm{d}} \cdot \frac{\mathrm{e}^{\mathrm{j} 6 n \omega_{1} t}+\mathrm{e}^{-\mathrm{j} 6 n \omega_{1} t}}{2} \\
& +\mathrm{j} \cdot \hat{i}_{\mathrm{s}, \mathrm{u} k}^{\mathrm{q}} \cdot \frac{\mathrm{e}^{\mathrm{j} 6 n \omega_{1} t}-\mathrm{e}^{-\mathrm{j} 6 n \omega_{1} t}}{2 \mathrm{j}} .
\end{aligned}
$$

Transforming the current into $\alpha \beta$-coordinates yields:

$$
\begin{aligned}
\vec{i}_{\mathrm{s}, \mathrm{u} k}^{\alpha \beta}= & =\mathrm{i}_{\mathrm{s}, \mathrm{u} k}^{\mathrm{dq}} \cdot \mathrm{e}^{\mathrm{j} \omega_{1} t} \\
= & \frac{1}{2} \hat{i}_{\mathrm{s}, \mathrm{u} k}^{\mathrm{d}} \cdot\left(\mathrm{e}^{\mathrm{j}(6 n+1) \omega_{1} t}+\mathrm{e}^{-\mathrm{j}(6 n-1) \omega_{1} t}\right) \\
& +\frac{1}{2} \hat{i}_{\mathrm{s}, \mathrm{u} k}^{\mathrm{q}} \cdot\left(\mathrm{e}^{\mathrm{j}(6 n+1) \omega_{1} t}-\mathrm{e}^{-\mathrm{j}(6 n-1) \omega_{1} t}\right) .
\end{aligned}
$$
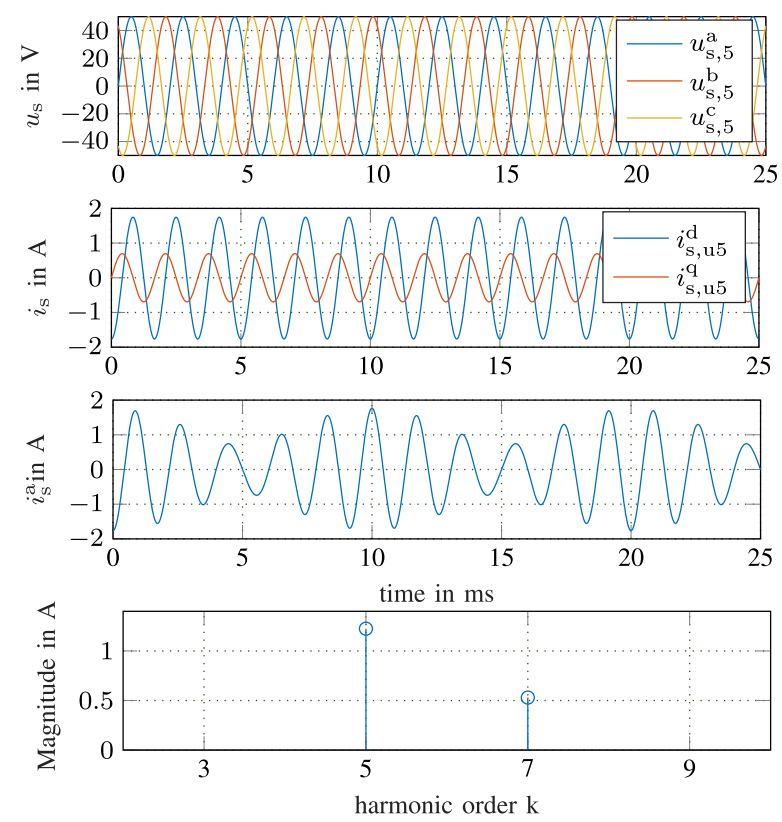

Fig. 4. Currents for an applied 5th order voltage and corresponding phase current spectrum.

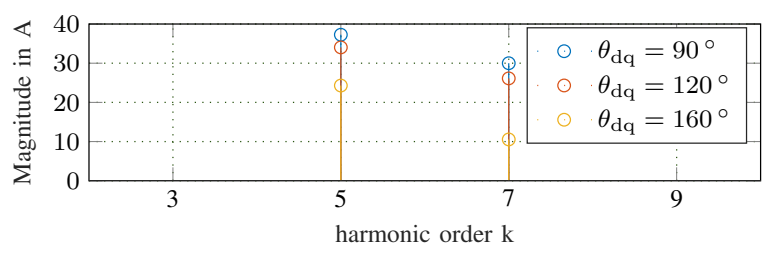

Fig. 5. Spectra of the harmonic phase currents for a variation of $\theta_{\mathrm{dq}}$.

Finally, the current of one phase is stated as:

$$
\begin{aligned}
i_{\mathrm{s}, \mathrm{u} k}^{\alpha}= & \Re\left\{\vec{i}_{\mathrm{s}, \mathrm{u} k}^{\alpha \beta}\right\}, \\
= & \frac{1}{2}\left(\hat{i}_{\mathrm{s}, \mathrm{u} k}^{\mathrm{d}}+\hat{i}_{\mathrm{s}, \mathrm{u} k}^{\mathrm{q}}\right) \cdot \cos \left((6 n+1) \omega_{1} t\right) \\
& +\frac{1}{2}\left(\hat{i}_{\mathrm{s}, \mathrm{u} k}^{\mathrm{d}}-\hat{i}_{\mathrm{s}, \mathrm{u} k}^{\mathrm{q}}\right) \cdot \cos \left((6 n-1) \omega_{1} t\right) .
\end{aligned}
$$

Equation 8 reveals that in case of $\hat{i}_{\mathrm{s}, \mathrm{u} k}^{\mathrm{d}}=\hat{i}_{\mathrm{s}, \mathrm{u} k}^{\mathrm{q}}$ the latter term becomes zero. Consequently, for non-salient machines, a harmonic voltage of order $k$ causes only a phase current of the same harmonic order.

To evaluate the coupling between the phase currents caused by related positive and negative sequence harmonic voltages, in a further step, two harmonic voltages of order $k=6 n \pm 1$ with $n=1$ are applied to the machine. The resulting current spectra for an exemplary set of different angles $\theta_{\mathrm{dq}}$ are shown in figure 5. It can be observed that the spectra and, therefore, the total harmonic content varies with $\theta_{\mathrm{dq}}$. Both negative and positive sequence harmonic voltages cause current components in d-and q-axis with the same frequency of $6 n \omega_{1}$ but with a different phase shift related to $\theta_{\mathrm{dq}}$. Consequently, the resulting magnitudes of the current components in the d- and q-axis vary as well leading to the effect which may be observed in figure 5 . 


\section{The Optimization Problem}

As a conclusion from the previous considerations, the load and trajectory dependent angle $\theta_{\mathrm{dq}}$ must be taken into account in the derivation of an appropriate cost function to minimize the total harmonic content of the phase currents of a salient synchronous machine. In the following, the full set of relevant equations for the derived optimization problem are stated.

First, the cost function is derived. The expressions for the harmonic currents are deduced by superposing the current components evoked by related voltage harmonics $k=6 n \pm 1$ which can be derived using the the set of equations given in (1), (4) and (5). A harmonic current of order $k$ in phase $a=\alpha$ can be formulated as in the following. For positive sequence harmonics, the current $i_{\mathrm{s}, k}^{\mathrm{a}}$ is stated as:

$$
\begin{aligned}
i_{\mathrm{s}, 6 n+1}^{\mathrm{a}}= & \left(P_{1 \mathrm{a}}+P_{2 \mathrm{a}}\right) \cdot \sin \left((6 n+1) \omega_{1} t\right) \\
& +\left(P_{1 \mathrm{~b}}+P_{2 \mathrm{~b}}\right) \cdot \cos \left((6 n+1) \omega_{1} t\right) .
\end{aligned}
$$

with the following definitions:

$$
\begin{aligned}
& P_{1 \mathrm{a}}=-\frac{1}{2 L_{\mathrm{sq}} \omega_{1}} \frac{\hat{u}_{\mathrm{s}, 6 \mathrm{n}+1}(\boldsymbol{\alpha})}{(6 n+1)} \cdot(\chi+1) \cdot \cos \left((6 n+1) \theta_{\mathrm{dq}}\right), \\
& P_{1 \mathrm{~b}}=-\frac{1}{2 L_{\mathrm{sq}} \omega_{1}} \frac{\hat{u}_{\mathrm{s}, 6 \mathrm{n}+1}(\boldsymbol{\alpha})}{(6 n+1)} \cdot(\chi+1) \cdot \sin \left((6 n+1) \theta_{\mathrm{dq}}\right), \\
& P_{2 \mathrm{a}}=\frac{1}{2 L_{\mathrm{sq}} \omega_{1}} \frac{\hat{u}_{\mathrm{s}, 6 \mathrm{n}-1}(\boldsymbol{\alpha})}{(6 n-1)} \cdot(\chi-1) \cdot \cos \left((6 n-1) \theta_{\mathrm{dq}}\right), \\
& P_{2 \mathrm{~b}}=\frac{1}{2 L_{\mathrm{sq}} \omega_{1}} \frac{\hat{u}_{\mathrm{s}, 6 \mathrm{n}-1}(\boldsymbol{\alpha})}{(6 n-1)} \cdot(\chi-1) \cdot \sin \left((6 n-1) \theta_{\mathrm{dq}}\right) .
\end{aligned}
$$

where $L_{\mathrm{sd}}=\frac{L_{\mathrm{sq}}}{\chi}$ is substituted. The corresponding negative sequence harmonic currents are derived in an analogous manner and are expressed as:

$$
\begin{aligned}
i_{\mathrm{s}, 6 n-1}^{\mathrm{a}}= & \left(N_{1 \mathrm{a}}+N_{2 \mathrm{a}}\right) \cdot \sin \left((6 n-1) \omega_{1} t\right) \\
& +\left(N_{1 \mathrm{~b}}+N_{2 \mathrm{~b}}\right) \cdot \cos \left((6 n-1) \omega_{1} t\right),
\end{aligned}
$$

with the following definitions:

$$
\begin{aligned}
& N_{1 \mathrm{a}}=\frac{1}{2 L_{\mathrm{sq}} \omega_{1}} \frac{\hat{u}_{\mathrm{s}, 6 \mathrm{n}-1}(\boldsymbol{\alpha})}{(6 n-1)} \cdot(\chi+1) \cdot \cos \left((6 n-1) \theta_{\mathrm{dq}}\right), \\
& N_{1 \mathrm{~b}}=\frac{1}{2 L_{\mathrm{sq}} \omega_{1}} \frac{\hat{u}_{\mathrm{s}, 6 \mathrm{n}-1}(\boldsymbol{\alpha})}{(6 n-1)} \cdot(\chi+1) \cdot \sin \left((6 n-1) \theta_{\mathrm{dq}}\right), \\
& N_{2 \mathrm{a}}=-\frac{1}{2 L_{\mathrm{sq}} \omega_{1}} \frac{\hat{u}_{\mathrm{s}, 6 \mathrm{n}+1}(\boldsymbol{\alpha})}{(6 n+1)} \cdot(\chi-1) \cdot \cos \left((6 n+1) \theta_{\mathrm{dq}}\right), \\
& N_{2 \mathrm{~b}}=-\frac{1}{2 L_{\mathrm{sq}} \omega_{1}} \frac{\hat{u}_{\mathrm{s}, 6 \mathrm{n}+1}(\boldsymbol{\alpha})}{(6 n+1)} \cdot(\chi-1) \cdot \sin \left((6 n+1) \theta_{\mathrm{dq}}\right)
\end{aligned}
$$

Figure 6 depicts the dependency of the magnitudes of the positive and negative sequence currents on $\theta_{\mathrm{dq}}$ calculated according (10) and (12). For a better visualization, the harmonic voltage excitation is of equal magnitude. The current components in figure 6 exhibit minima at all even multiples of $\theta_{\mathrm{dq}}=90^{\circ}$ and maxima at all odd multiples of $\theta_{\mathrm{dq}}=90^{\circ}$. The location of these extrema is independent of the order $k$. Therefore, the resulting distortion current exhibits minima and maxima at the same values of $\theta_{\mathrm{dq}}$.

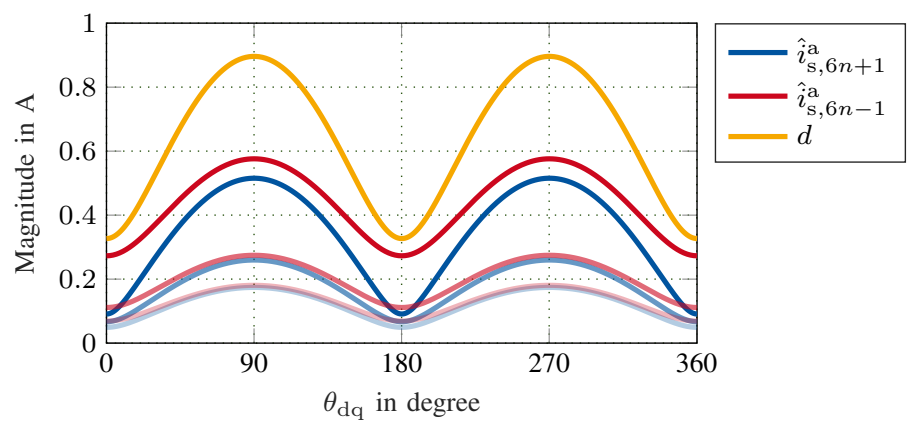

Fig. 6. Positive and negative sequence currents for $n=1,2,3$ and resulting distortion.

Note that for the special case of a non-salient machine, i.e., $\chi=1$, the negative sequence components $P_{2 \mathrm{a}}$ and $P_{2 \mathrm{~b}}$ vanish, cf. (10). The same applies for $N_{2 \mathrm{a}}$ and $N_{2 \mathrm{~b}}$. Therefore, the cross coupling between positive and negative sequence harmonics is only present for machines with saliency. The equations (9) and (11) lead to magnitudes of the positive and negative sequence harmonic phase currents as in (13).

$$
\begin{aligned}
& \hat{i}_{\mathrm{s}, 6 n+1}^{\mathrm{a}}=\sqrt{\left(P_{1 \mathrm{a}}+P_{2 \mathrm{a}}\right)^{2}+\left(P_{1 \mathrm{~b}}+P_{2 \mathrm{~b}}\right)^{2}} \\
& \hat{i}_{\mathrm{s}, 6 n-1}^{\mathrm{a}}=\sqrt{\left(N_{1 \mathrm{a}}+N_{2 \mathrm{a}}\right)^{2}+\left(N_{1 \mathrm{~b}}+N_{2 \mathrm{~b}}\right)^{2}}
\end{aligned}
$$

A nonlinear constraint is due to the definition of the modulation index and a set of linear constraints to guarantee that the switching angles are ordered and do not exceed the quarter period.

This yields the following optimization problem:

$$
\begin{array}{ll}
\min _{\boldsymbol{\alpha}} & d=\sqrt{\sum_{n=1}^{\infty}\left(\hat{i}_{\mathrm{s}, 6 n \pm 1}^{\mathrm{a}}(\boldsymbol{\alpha})\right)^{2}} \\
\text { subject to } \quad m & =\frac{4}{\pi} \cdot \sum_{i=1}^{M}(-1)^{(i+1)} \cos \left(\alpha_{i}\right) \\
& 0 \leq \alpha_{1} \leq \alpha_{2} \leq \cdots \leq \alpha_{M} \leq \frac{\pi}{2} .
\end{array}
$$

Observe that the equations in (10) and (12) contain the same factor $\frac{1}{2 L_{\mathrm{sq}} \omega_{1}}$. Defining $\hat{i}_{\mathrm{s}, 6 n \pm 1}^{\mathrm{a}}(\boldsymbol{\alpha})^{\prime}=2 L_{\mathrm{sq}} \omega_{1} \hat{i}_{\mathrm{s}, 6 n \pm 1}^{\mathrm{a}}(\boldsymbol{\alpha})$, the cost function can be written as:

$$
d=\frac{1}{2 L_{\mathrm{sq}} \omega_{1}} \sqrt{\sum_{n=1}^{\infty}\left(\hat{i}_{\mathrm{s}, 6 n \pm 1}^{\mathrm{a}}(\boldsymbol{\alpha})^{\prime}\right)^{2}} .
$$

Since this factor has only scaling effect on the cost function, the optimal switching angles $\alpha$ of (14) are independent of the fundamental angular frequency $\omega_{1}$ and of $L_{\mathrm{sq}}$. Furthermore, in case of a non-salient machine $(\chi=1)$, the equations in (13) may be simplified as:

$$
\begin{aligned}
\hat{i}_{\mathrm{s}, 6 n+1}^{\mathrm{a}}= & \sqrt{P_{1 \mathrm{a}}^{2}+P_{1 \mathrm{~b}}^{2}}, \\
= & \frac{1}{L_{\mathrm{s}} \omega_{1} \frac{\hat{u}_{\mathrm{s}, 6 \mathrm{n}+1}(\boldsymbol{\alpha})}{(6 n+1)}} \\
& \cdot \underbrace{\sqrt{\cos \left((6 n+1) \theta_{\mathrm{dq}}\right)^{2}+\sin \left((6 n+1) \theta_{\mathrm{dq}}\right)^{2}}}_{=1} .
\end{aligned}
$$


The computation for $\hat{i}_{\mathrm{s}, 6 n-1}^{\mathrm{a}}$ follows analogously and is omitted here.

Note that the harmonic currents are independent of the operating point dependent angle $\theta_{\mathrm{dq}}$ in case of non-salient machines. Normalizing the harmonic currents on the fundamental current of an inductor $i_{\mathrm{s}, 1}^{\mathrm{a}}=\frac{\hat{u}_{\mathrm{s}, 1}(\alpha)}{\omega_{1} L_{\mathrm{s}}}$ yields the definition of the weighted total harmonic distortion (WTHD):

$$
\mathrm{WTHD}=\frac{\sqrt{\sum_{n=1}^{\infty}\left(\frac{\hat{u}_{\mathrm{s}, 6 n \pm 1}(\boldsymbol{\alpha})}{6 n \pm 1}\right)^{2}}}{\hat{u}_{\mathrm{s}, 1}(\boldsymbol{\alpha})} .
$$

The WTHD is commonly used as a cost function to determine OPPs for induction machines or synchronous machines (e.g. $\left.{ }^{(3)},{ }^{(10)}\right)$. In this section it is demonstrated that the approach of minimizing the WTHD is consistent with the deduced cost function for salient machines in (14).

\section{Comments on the Optimization Algorithm}

The optimization problem in (14) is solved numerically. It is highly nonlinear due to the trigonometric expressions in the cost function (14a) and the constraint (14b). These nonlinearities arise from the definition of the harmonic voltage magnitude in (1) and the constraint (14b) which is based on (1). In particular, sine and cosine feature many local minima which pushes standard optimization methods to their limits. Within this work, the commercial implementation of sequential quadratic programming (SQP) in Matlab's fmincon is used to solve the optimization problem in (14).

The SQP method was originally introduced in ${ }^{(11)}$ and computes reliably local minima of convex optimization problems, i.e., minimization problems with convex cost function and convex feasible sets. It is based on the sequential approximation of the optimization problem by a quadratic model. This means beginning with an initial guess, a local quadratic approximation of the cost function is obtained by Taylor expansion around the initial guess. Similarly, the constraints are linearized around that initial guess. This yields a socalled quadratic optimization problem for which highly efficient tools are available making the solvings of these fast. The solution of the approximating quadratic problems serves as the new iterate. A new quadratic model of the cost function and the linearization of the constraints is created around this iterate. This procedure is repeated until a convergence threshold is reached.

The SQP method is especially efficient if the number of variables is relatively small, as we have it in (14), since the Fourier decomposition is considered up to $M$ which results in $M$ decision variables, namely $\boldsymbol{\alpha}$. Usually, implementations of the SQP method include low rank updates on the Hessian of the cost function to reduce computational effort. For a general overview of this method and other optimization methods, please refer to ${ }^{(12)}$.

Clearly, the optimization problem (14) is not globally convex, however SQP finds a local minimum. In general, the application of locally convergent methods is very sensitive to the choice of the initial guess. Therefore, a globalization strategy is pursued where the optimization problem in (14) is solved for 1000 initial guesses for fixed modulation index $m$, saliency $\chi$ and angle $\theta_{\mathrm{dq}}$ and the best solution is selected

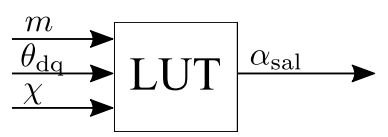

(a) Salient machines

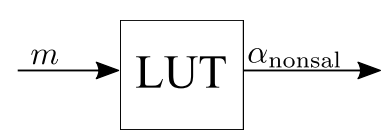

(b) Nonsalient machines

Fig. 7. Lookup tables for the optimized switching angles and respective input parameters.

at the end. The optimization problem is dependent on $m, \theta_{\mathrm{dq}}$ and $\chi$ which form a three-dimensional parameter space that is discretized by an equidistant grid. For each set $\left(m, \theta_{\mathrm{dq}}, \chi\right)$, one optimization problem must be solved. Problems with neighboring parameters in this grid are initialized by previously derived solutions in order to accelerate computations. This procedure also helps to avoid discontinuities in the optimal switching angles $\alpha_{i}$.

\section{Dependency of the optimization results on op- erating point dependent parameters}

The equations (10), (12) and (14) reveal that the optimization problem for a salient machine depends on two more parameters than for the non-salient case, namely the angle $\theta_{\mathrm{dq}}$ and the saliency $\chi$ which are both operating point dependent parameters. The angle $\theta_{\mathrm{dq}}$ depends on the control and the chosen operating trajectory while the saliency $\chi$ changes due to different saturation behavior of the $\mathrm{d}$ - and the q-axis inductances. Usually, for the case of permanent magnet machines, $L_{\text {sd }}$ saturates already at lower currents in the respective axis than $L_{\mathrm{sq}}$. That leads to a varying saliency depending on the applied current vector. The two additional dependencies increase not only the computational effort of the optimization problem but also the memory required for the lookup tables storing the offline calculated switching angles (figure 7). Many former studies (e.g. ${ }^{(3)(5)}$ ) show that the sets of optimized switching angles exhibit discontinuities over the modulation index.

However, using lookup tables and interpolation, these discontinuities lead to invalid or suboptimal sets of switching angles in case of interpolating around a discontinuity. Furthermore, if the operating point slightly varies around a discontinuity, temporary distortions of the volt-second balance can occur. These may lead to a significant dc offset in the currents if the control is not able to compensate this volt-second error fast enough. Therefore, these discontinuities should be avoided a priori as e.g. proposed in ${ }^{(3)}$.

In the following, it is investigated whether the additional parameters aggravate the issue of discontinuities. For this purpose, the optimization results are calculated by varying the parameters $m, \theta_{\mathrm{dq}}$ and $\chi$. As an example, figure 8 depicts the optimization results for the first switching angle $\alpha_{1}$ for a number of decision variables $M=5$ and a fixed saliency of $\chi=3$ varying the modulation index $m$ and $\theta_{\mathrm{dq}}$. Along the modulation index, discontinuities of the switching angles can be observed (sharp color changes). However, along the angle $\theta_{\mathrm{dq}}$ nearly no further discontinuities arise. Instead, the same discontinuities over $m$ shift at different values of $\theta_{\mathrm{dq}}$.

A very similar phenomenon occurs when varying the saliency at a fixed angle $\theta_{\mathrm{dq}}$, which is shown in figure 9 . Along the $\chi$-axis at a fixed modulation index, the optimal $\alpha_{1}$ 


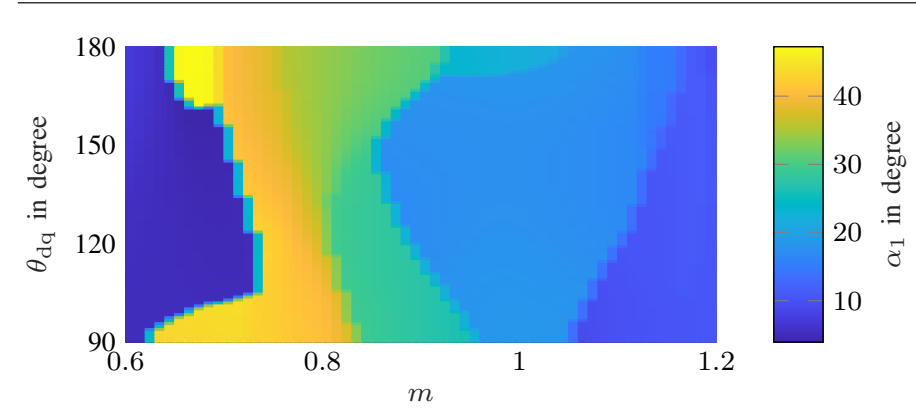

Fig. 8. Optimization results for $\alpha_{1}$ plotted over the modulation index $\mathrm{m}$ and $\theta_{\mathrm{dq}}$ for a fixed saliency $\chi=3$.

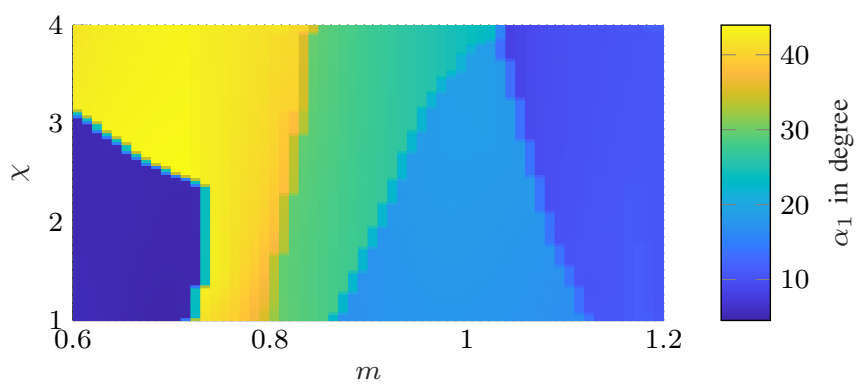

Fig. 9. Optimization results for $\alpha_{1}$ plotted over the modulation index $\mathrm{m}$ and $\chi$ for a fixed $\theta_{\mathrm{dq}}=90^{\circ}$.

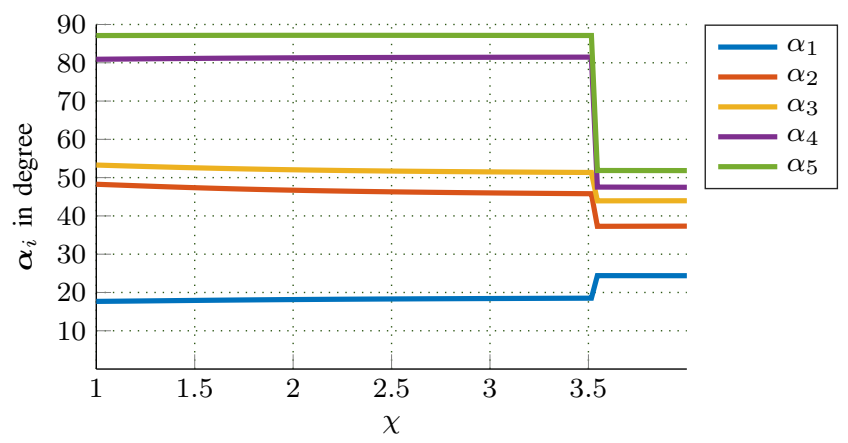

Fig. 10. Optimization results $\alpha$ for a fixed $\theta_{\mathrm{dq}}=90^{\circ}$ and $m=0.9$.

profile is relatively smooth. The low sensitivity of the optimal switching angles on the saliency is further illustrated in figure 10, in which the entire set of decision variables $\boldsymbol{\alpha}$ is plotted over the saliency for a fixed $m$ and $\theta_{\mathrm{dq}}$. For a wide range of saliency, the results are smooth. Even if the saliency varies by one integer number, the respective switching angles change less than $3^{\circ}$. This shows that the increase of complexity due to two additional parameters does not necessarily result in more discontinuous solutions.

\section{Benchmark with conventional Optimization Approach for nonsalient machines}

In this section, the proposed optimization approach is compared to the conventional WTHD approach. For the benchmark, the relative deviation in distortion is introduced as:

$$
r_{\mathrm{d}}=\frac{d\left(\boldsymbol{\alpha}_{\text {nonsal }}\right)-d\left(\boldsymbol{\alpha}_{\text {sal }}\right)}{d\left(\boldsymbol{\alpha}_{\text {nonsal }}\right)} .
$$

where $\boldsymbol{\alpha}_{\text {nonsal }}$ is computed according to (17) as cost function and the same constraints as defined in (14) and $\boldsymbol{\alpha}_{\text {sal }}$ represents the optimal switching angles computed according to the saliency considering optimization problem stated in (10),

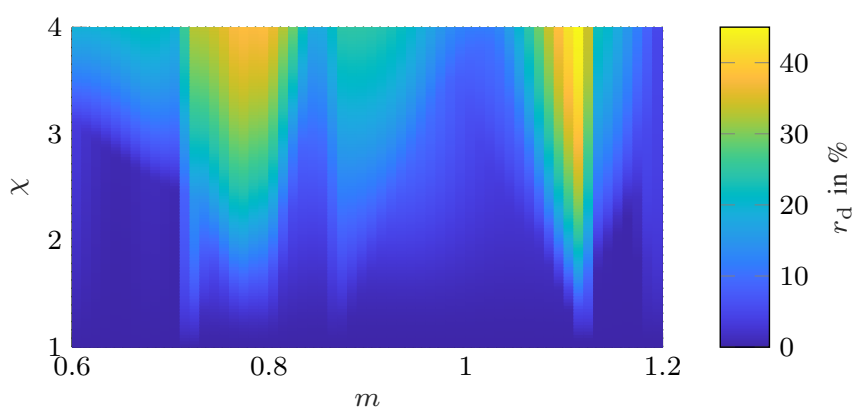

Fig. 11. Relative deviation in distortion comparing the salient with the nonsalient optimization approach for different saliencies and modulation indices at a fixed angle $\theta_{\mathrm{dq}}=90^{\circ}$.

(12) and (14). Note that (18) delivers the same ratio as for the THD of the phase currents. The two optimizations to obtain $\boldsymbol{\alpha}_{\text {nonsal }}$ and $\boldsymbol{\alpha}_{\text {sal }}$ are both performed with the SPQ algorithm applying the same number of initial guesses. The distortion $d$ is calculated analytically based on the set of equations given in (10), (12) and (14). Figure 11 depicts the results of the benchmark over a range of modulation indices and saliencies at a fixed angle $\theta_{\mathrm{dq}}$.

The correlation of the reduction of distortion current with the saliency is clearly visible. For nonsalient machines $(\chi=$ 1) both methods deliver optimal switching angle sets which lead to the same distortion. With increasing saliency, the deduced method outperforms the conventional WTHD based method.

The same benchmark is performed varying the angle $\theta_{\mathrm{dq}}$ at a fixed saliency as shown in figure 12. The largest deviation $\Delta d$ occurs at $\theta_{\mathrm{dq}}=90^{\circ}$ and $\theta_{\mathrm{dq}}=180^{\circ}$ while the lowest deviation occurs at $\theta_{\mathrm{dq}}=135^{\circ}$. Note that for $\theta_{\mathrm{dq}}=(2 n-1) \cdot 45^{\circ}$ $\left|\cos \left((6 n \pm 1) \theta_{\mathrm{dq}}\right)\right|=\left|\sin \left((6 n \pm 1) \theta_{\mathrm{dq}}\right)\right|=\frac{\sqrt{2}}{2}$. With this relation the objective function in (14) can be simplified to

$$
d=\frac{1}{\sqrt{2} L_{\mathrm{sq}} \omega_{1}} \cdot \sqrt{\chi^{2}+1} \cdot \sqrt{\sum_{n=1}^{\infty}\left(\frac{\hat{u}_{\mathrm{s}, 6 \mathrm{n} \pm 1}(\boldsymbol{\alpha})}{6 n \pm 1}\right)^{2}} .
$$

Except for a constant scalar, the expression in (19) is equivalent with the nominator of the WTHD in (17). However, these constant scalars have no impact on the optimization results which are in this case the optimal switching angles $\alpha$. Due to the same optimal $\alpha$ (see figure 14), no deviation in distortion $r_{\mathrm{d}}$ at $\theta_{\mathrm{dq}}=(2 n-1) \cdot 45^{\circ}$ can be observed for the benchmark with the conventional approach. The results of $r_{\mathrm{d}}$ also vary with the modulation index, as it can been observed in 11 and 12 respectively. Figure 13 depicts the sets of optimal switching angles over the modulation index for a specific $\theta_{\mathrm{dq}}$ and $\chi$ and reveals that the optima of both objective functions nearly coincide for some modulation index ranges.

\section{Conclusion}

This paper proposes an approach to compute quarter wave symmetric optimized pulse patterns for machines with saliency. It is demonstrated that especially at large saliencies, the deduced approach outperforms the commonly used approach for nonsalient machines. Moreover, numerical results indicate that the sensitivity of the computed optimal switch- 


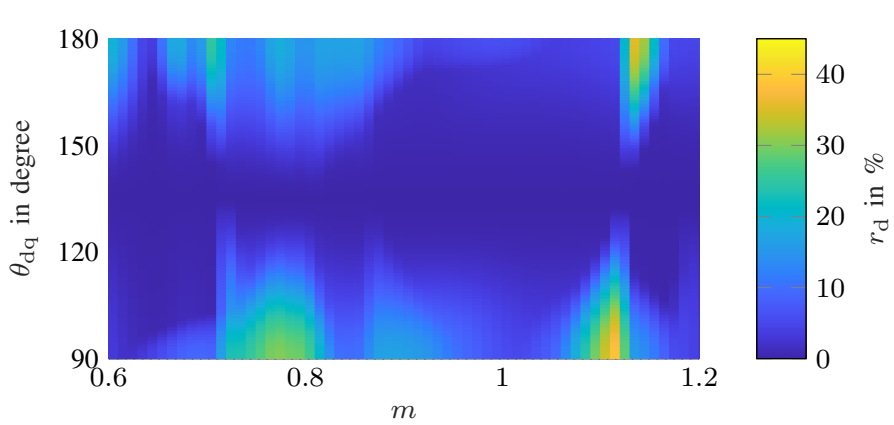

Fig. 12. Relative deviation in distortion comparing the salient with the nonsalient optimization approach for different angles $\theta_{\mathrm{dq}}$ and modulation indices at a fixed saliency $\chi=3$.

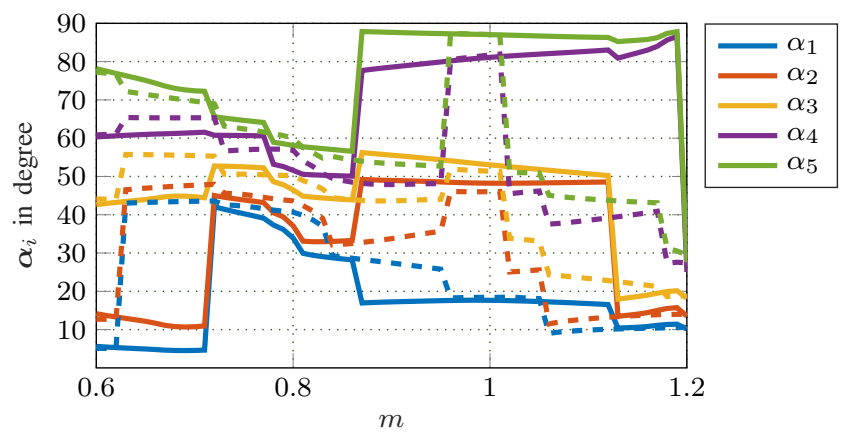

Fig. 13. Optimization results $\alpha$ for a fixed $\theta_{\mathrm{dq}}=90^{\circ}$ and $\chi=3$. The dotted lines respresent the optimization results according to (14).

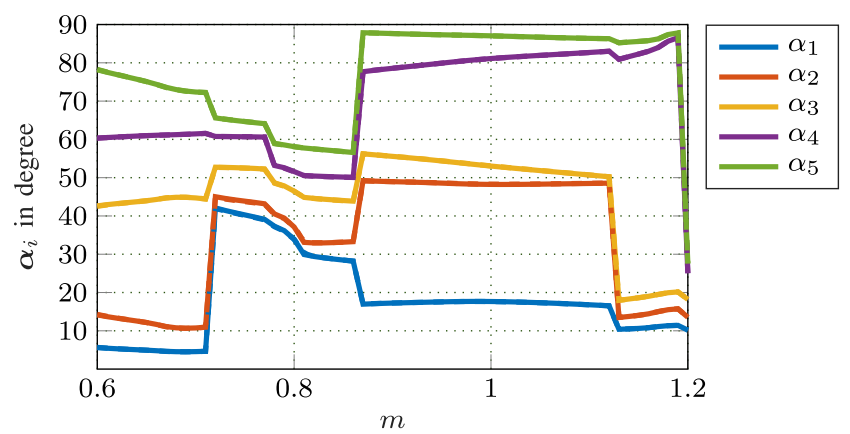

Fig. 14. Optimization results $\alpha$ for a fixed $\theta_{\mathrm{dq}}=135^{\circ}$ and $\chi=3$. The dotted lines respresent the optimization results according to (14).

ing angles to the saliency is relatively low and does not necessarily lead to further discontinuities in the computed sets of switching angles.

The abolishment of quarter wave symmetry increases the potential to reduce the THD for salient machines even further $\left(\mathrm{see}^{(13)}\right)$. Therefore, future work focuses on efficient computational strategies to obtain optimal and smooth solutions for a reasonable operating range despite the increased problem complexity due to larger sets of decision variables in half wave symmetric optimized pulse patterns.

\section{References}

( 1 ) N.Hartgenbusch, A. Thünen, R.W. De Doncker: "Optimized Pulse Patterns for Salient Synchronous Machines", International Conference on Electrical Machines and Systems (ICEMS), 2020
( 2 ) G. S. Buja, G. B. Indri: "Optimal Pulsewidth Modulation for Feeding AC Motors", IEEE Transactions on Industry Applications, pp. 38-44 (1977)

( 3 ) A. K. Rathore, T. Boller, J. Holtz: "Generalized Optimal Pulsewidth Modulation of Multilevel Inverters for Low-Switching-Frequency Control of Medium-Voltage High-Power Industrial AC Drives", IEEE Transactions on Industrial Electronics, pp. 4215-4224 (2013)

( 4 ) T. Geyer: "Model Predictive Control of High Power Converters and Industrial Drives", Wiley, 2017

( 5 ) K. Peter, J. Böcker, F. Mink, S. Beineke: "Comparison of quarter-wave with half-wave symmetrical pulse patterns applied in electrical high-speed drives", 8th IET International Conference on Power Electronics, Machines and Drives (PEMD), 2016

( 6 ) J.E.Huber, A. J. Korn: "Optimized Pulse Pattern Modulation for Modular Multilevel Converter High-Speed Drive", 15th International Power Electronics and Motion Control Conference (EPE/PEMC), 2012

( 7 ) F. Mink, K. Peter, H. Kasten, S. Beineke: "Feedback control of high-speed PMSM with synchronous optimal PWM", 18th European Conference on Power Electronics and Applications (EPE'16 ECCE Europe), 2016

( 8 ) Z. Zhang, J. Xu: "Closed-Loop Control of PMSM Based on Optimal Synchronous Pulse Patterns", International Power Electronics and Application Conference and Exposition, 2014

( 9 ) J. Holtz, G. da Cunha, N. Petry, P. José Torri: "Control of Large Salient-Pole Sychronous Machines Using Synchronous Optimal Pulsewidth Modulation", IEEE Transactions on Industrial Electronics, pp.3372-3379 (2015)

(10) G. Holmes, T. Lipo: "Pulse Width Modulation for Power Converters", Wiley, 2003

(11) R.B. Wilson: "A simplicial algorithm for concave programming", Dissertation, 1963

(12) J. Nocedal, S. Wright: "Numerical Optimization", Springer Verlag GmbH, 2006

(13) A. D. Birda, J. Reuss, C. Hackl: "Synchronous optimal pulse-width modulation with differently modulated waveform symmetry properties for feeding synchronous motor with high magnetic anisotropy", 2017 19th European Conference on Power Electronics and Applications (EPE'17 ECCE Europe), 2017

Nina Hartgenbusch (Non-member) received the B.Sc. and M.Sc.

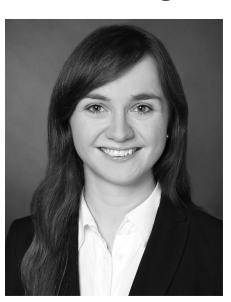
Electrical Engineering from the Faculty of Electrical Engineering, Information Technology and Computer Engineering, RWTH Aachen University, Aachen, Germany, in 2015 and 2018, respectively. Since 2018, she is a Research Associate with the Institute for Power Electronics and Electrical Drives (ISEA) headed by Prof. Rik De Doncker, RWTH Aachen University. She is currently working towards her $\mathrm{PhD}$ with focus on innovative modulation schemes for traction drive inverters. Moreover, her research interests include predictive control algorithms for permanent magnet synchronous machines.

Anna Thünen (Non-member) received the B.Sc. and M.Sc. in Math-

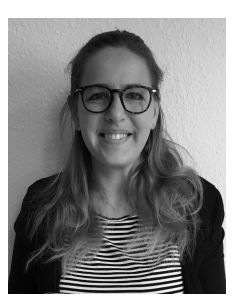
ematics with specialisation in optimization and numerics from Otto-von-Guericke University in Magdeburg. In 2017, she joined the Institute for Geometry and Practical Mathematics at RWTH Aachen University in the research group continuous optimization headed by Prof. Michael Herty. In 2021, she is awarded the $\mathrm{PhD}$ in mathematics for her dissertation on Nash equilibria in multi-level games. Her research focuses on complex structures in optimization and optimal control, especially non smooth non convex problems arising in multi-level or mixed-integer models. Applications include energy markets, traffic on networks, and engineering. Besides, she is an active member of the Society of Industrial and Applied Mathematics (SIAM) and was awarded the SIAM certificate of recognition in 2020. 
Rik W. De Doncker (Non-member) received the Ph.D. degree in elec-

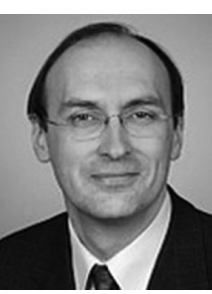
trical engineering from Katholieke Universiteit Leuven, Leuven, Belgium, in 1986. In 1987, he was appointed as a Visiting Associate Professor at the University of Wisconsin, Madison. After a short stay as an Adjunct Researcher with Interuniversity Microelectronics Centre, Leuven, he joined, in 1989, the Corporate Research and Development Center, General Electric Company, Schenectady, NY, USA. In 1994, he joined Silicon Power Corporation, a former division of General Electric, Inc., as the Vice President of Technology. In 1996, he became a Professor at RWTH Aachen University, Aachen, Germany, where he currently leads the Institute for Power Electronics and Electrical Drives (ISEA). Since 2006, he has been the Director of the E.ON Energy Research Center, RWTH Aachen University. He has authored more than 400 technical papers and is holder of more than 40 patents. Dr. De Doncker was the President of the IEEE Power Electronics Society (PELS) in 2005 and 2006. He was the founding Chairman of the German IEEE Industry Applications Society PELS Joint Chapter. In 2002, he was the recipient of the IEEE IAS Outstanding Achievement Award. In 2008, he received the IEEE PES Nari Hingorani Custom Power Award. In 2009, he led a VDE/ETG Task Force on Electric Vehicles. In 2010, he received an Honorary Doctor degree of TU Riga, Riga, Latvia. In 2013, he received the IEEE William E. Newell Power Electronics Award. In 2020, he received the IEEE Medal in Power Engineering. 